\title{
Length of hospital stay following hip fracture and risk of 30 and 90 day mortality in a United Kingdom cohort
}

\author{
Hassaan Q. Sheikh, Amro Alnahhal, Adeel Aqil, Fahad S. Hossain \\ From the Leeds General Infirmary, Great George Street, Leeds, LS1 3EX, United Kingdom.
}

\begin{abstract}
A recent study identified a length of stay (LOS) of 10 days or less following hip fracture is associated with increased risk of 30-day mortality. This effect has not been previously studied for 90-day mortality or in the United Kingdom (UK). Our aim was to investigate the effect of LOS on 30-day and 90-day mortality following hip fracture in a UK population. In this single-centre study, we retrospectively identified consecutive patients with a hip fracture from the National Hip Fracture Database over 3 years. We excluded patients who died as inpatient during their index admission. The main end-points were 30-day and 90-day mortalities and risk factors for these were examined using stepwise univariate and multivariate Cox regression analyses. Of 1228 patients, mortality at 30-days was $1.2 \%$ (15 patients) and $7.8 \%$ (96 patients) at 90-days. Mean LOS was 24 days and a total of 206 patients had a LOS of 10 days or less. Following cariate analysis, the highest risk factor for 30-day mortality was a LOS of 10 days or less with a hazard ratio of 7.26 (95\% confidence interval 2.56-20.51). Other risk factors for 30-day mortality included increasing age, male gender and chest infection. A LOS of 10 days or less was not associated with increased mortality at 90-days. A short LOS was associated with higher risk of early mortality. We recommend that hip fracture patients be fully rehabilitated prior to discharge to reduce the risk of early mortality.
\end{abstract}

Keywords: length of stay; hip fracture; proximal femur fracture; epidemiology; mortality.

No benefits or funds were received in support of this study. None of the authors have a conflict of interest.

\section{INTRODUCTION}

It is well established that fragility hip fractures carry a poor prognosis with high early mortality and long term sequelae of reduced mobility, chronic pain and loss of independence (1). The total annual hospital costs related to hip fracture management is estimated to be in excess of $£ 1$ billion to the British healthcare system. $61 \%$ of this cost is related to the index hospital admission (2). The direct fiscal burden of admission and subsequent inpatient stay after a hip fracture may therefore incentivise hospitals to expedite discharge of these patients as soon as it is safe to do so in order to minimise expenditure. From a management perspective it helps improve efficiency of healthcare delivery by minimising bed occupancy while improving patient flow and has

\footnotetext{
Hassaan Q. Sheikh',

Amro Alnahhal²,

Adeel Aqil ${ }^{1}$,

- Fahad S. Hossain ${ }^{3}$

${ }^{1}$ Leeds General Infirmary, Great George Street, Leeds, LS1

3EX, United Kingdom.

${ }^{2}$ Barnsley Hospital, Gawber Road, Barnsley, S75 3EP, United Kingdom.

${ }^{3}$ Walsall Helathcare NHS trust, Moat road, Walsall, WS2 9Ps, United Kingdom.

Correspondence : Hassaan Sheikh Northern General Hospital, Herries Road, Sheffield, S5 7AU, United Kingdom.

Email : hqsheikh@doctors.org.uk

- 2021, Acta Orthopædica Belgica.
} 
become a dominant theme of the NHS Improvement framework.

In contrast however, there is concern that shorter LOS may have the unintended consequence of poorer patient outcomes. With limited resources and financial pressures, it is possible that some patients are discharged early from hospitals leading to the occurrence of adverse events. A previous study of 59 Norwegian hospitals has shown that shortened LOS resulted in an increase in the risk of early unplanned readmission (3) while another study showed an association with increased risk of post discharge mortality in a large cohort of patients admitted to hospital with acute pulmonary embolism (4). Previous studies examining the association between LOS and patient outcomes such as mortality have been confounded by patient-level factors. In particular it has been suggested that LOS is closely related to disease severity with patients of shorter LOS being inherently less severe in disease profile compared to those of longer hospital stays. As such, observational studies comparing outcomes between short and long LOS are perhaps biased towards finding favourable outcomes for patients of shorter LOS, consequently failing to show a true effect owing to patient level confounding factors (5).

It is well established that early mortality after hip fracture management is due to medical causes such as respiratory infections or cardiac events (6-9). Such fragility hip fracture patients are generally frailer compared to those of other hospital admissions. Previous large cohort and registry based studies have shown that around two-thirds of all fragility hip fracture patients have an ASA grade of 3 or more $(7,8,10)$. Higher ASA grades due to more severe co-morbidity profile are inevitably associated with increased early mortality. For any study analysing mortality risk factors, however, it is imperative that specific diagnoses are examined alongside other potential risk factors such as LOS.

A large registry level study from Sweden identified that inpatient stay of 10 days or less following a hip fracture is associated with an elevated risk of 30-day mortality (11). The authors included 116,111 patients and analysed 30-day post-discharge mortality. They also found that each reducing day of stay less than 10 days increased the risk of 30-day mortality by up to $16 \%$ and the most significant risk factor of increased mortality was length of stay (LOS) less than five days. A length of stay (LOS) over 10 days had no effect on mortality. The authors were not able to analyse for causes of mortality in this cohort. Similar findings have been reported by another study comparing mean LOS for hip fractures between Japanese and US populations, concluding that increasing LOS is associated with reduction in early mortality (12). This trend is perhaps of significance within the setting of the United Kingdom National Health Service (NHS) where reducing LOS is prioritised as a means to improving patient outcomes and efficiency of healthcare delivery (13).

The aim of this study therefore was to ascertain whether a shorter LOS affects 30-day and 90-day mortality in a UK fragility hip fracture population.

\section{MATERIALS AND METHODS}

STROBE criteria were followed throughout the design, data collection, analysis and write-up of this study. We retrospectively collected data taken from the National Hip Fracture Database (NHFD, England and Wales) between September 2008 and March 2011 following institutional board approval from a single centre. All consecutive hip fracture patients presenting to a single Major Trauma Centre (Leeds General Infirmary) in the UK were identified. Data at our centre is collected prospectively by specially trained data clerks. Data analysed included patient demographics (including age, gender, pre-injury walking ability and admission source), preoperative blood test parameters, medical comorbidities, fracture type and American Society of Anesthesiologists (ASA) grade. Fractures were classified as intracapsular undisplaced, intracapsular displaced, intertrochanteric, subtrochanteric or other (if the injury couldn't be distinctively classified).

Blood test parameters included haemoglobin levels, white cell count, coagulation profile, urea and electrolyte levels. Patients' preoperative admission source was categorised in line with the NHFD categories of patients who were independent in their home; patient residing in a care home such 
as a residential or nursing home; patients who were already an inpatient at the time of injury; and patients that were transferred from a different hospital after injury. Patient pre-injury walking ability was categorised as independent ambulators; ambulators with a single stick/crutch; patients requiring two or more sticks/crutches for ambulation and non ambulators who used a wheel chair for mobility.

The Charlson co-morbidity index was calculated using International Classification of Disease, 10th revision (ICD-10) codes as classified in our inpatient database (14). Other data collected included time to surgery from initial presentation and hospital length of inpatient stay in days. Our primary endpoint of interest was the incidence of 30-day mortality (calculated from the time of admission). We also collected causes of 30-day mortality from death certificate entries as well as from coroner's autopsy records. Our secondary endpoint was incidence of 90day mortality. We excluded patients with duplicate and incomplete records. Furthermore, in an effort to reduce patient level confounding we excluded any patients who died as an inpatient during their index admission. This is because any patients that died as inpatients will have a LOS directly linked to their death and therefore it is difficult to analyse LOS as a risk factor for the death itself.

All patients were treated according to a standardised hip fracture protocol based on national guidance (15). On admission all patients received full clinical assessment for fitness for surgery including a clinical exam, ECG, chest plain x-ray radiograph and a full blood haematological, biochemical and clotting profile. We aimed to perform surgery within 36 hours of initial presentation. The surgical intervention for each patient was decided following a multidisciplinary team review (consisting of orthopaedic surgeons, anaesthetists, ward and therapy staff). All procedures were performed in a laminar flow theatre under supervision of an orthopaedic consultant. Displaced intracapsular fractures were treated with a hip hemiarthroplasty, whereas stable and undisplaced intracapsular fractures were treated with in-situ fixation. Intertrochanteric fractures were treated with a dynamic hip screw and subtrochanteric fractures with a cephalomedullary nail. Patients who were not cognitively impaired and mobile independently without or with the use of a single stick received a total hip arthroplasty. Following the operation, we aimed to mobilise all patients fully weight bearing immediately post operatively.

As the Swedish study (11) had found a LOS cutoff point of 10 days or less to be significant when predicting mortality, we used the same cut-off in our cohort to divide LOS into a dichotomous independent variable. We analysed all collected variables against the risk of mortality at 30-days and 90-days on univariate analysis using a chisquared/Fisher exact test for categorical data or the independent $\mathrm{t} / \mathrm{Mann}$-Whitney U-test for continuous variables. The impact of LOS on 30-day and 90day mortality was then assessed using a backward stepwise multiple regression model while adjusting for covariates. Covariates were included in the regression model if the univariate analysis resulted in a p-value of $<0.15$, in accordance with accepted and published statistical methods (16). All statistical calculations were performed using IBM SPSS software, version 21 (Armonk, New York, USA).

\section{RESULTS}

Out of a total of 1673 patients initially identified from the NHFD, following exclusions (as described in methods) 1228 were finally included in the analysis (figure 1). Mean age of patients was 81 years and the majority of patients (908 patients) were female. Mortality at 30-days was $1.2 \%$ (15 patients) and 7.8\% (96 patients) at 90-days. LOS was not normally distributed and median LOS was 18 days. $16.8 \%$ (206) of patients had a LOS of 10 days or less. At 30-days the commonest overall causes of death was pneumonia followed by sepsis of other origin. Causes of death beyond 30-days were not available for analysis. Causes of death separated by LOS are summarised in figure 2 .

Tables 1-4 provide the univariate analysis of risk factors for mortality at 30-days and 90-days. Univariate analysis showed that the proportion of patients with male gender, increasing age and LOS of $<10$ days were significantly higher in the 30-day mortality group. Similarly, in the 90-day mortality cohorts, patients were likely to be older and have 


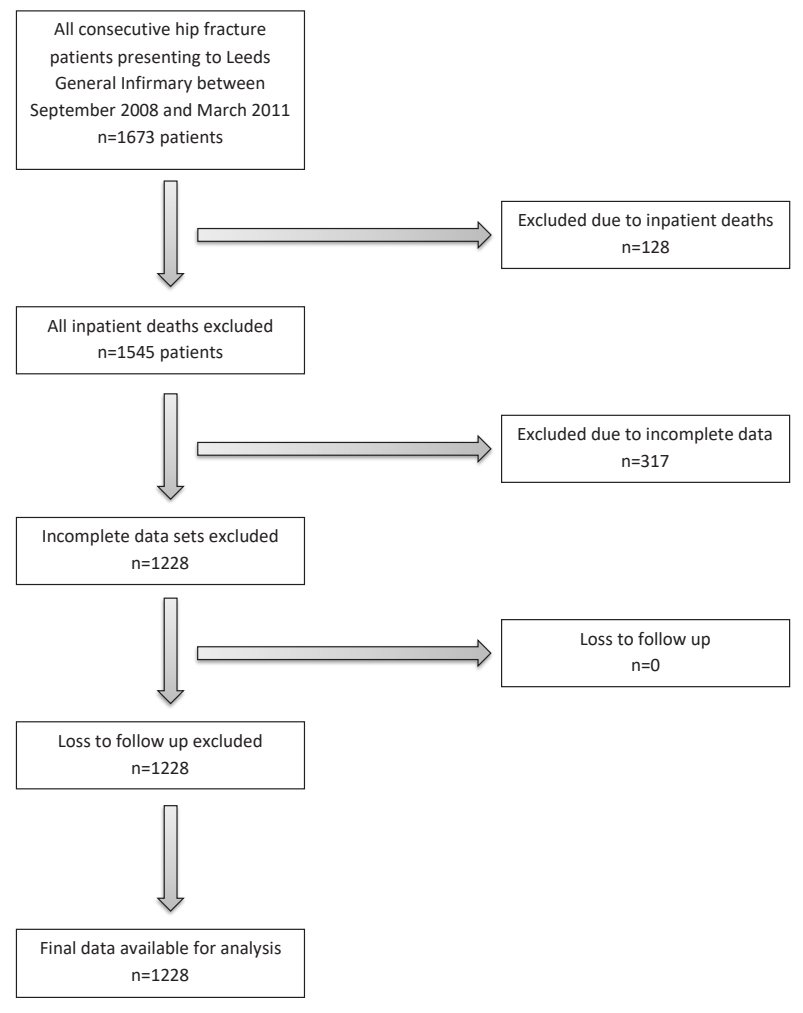

Figure 1. - STROBE flowchart.
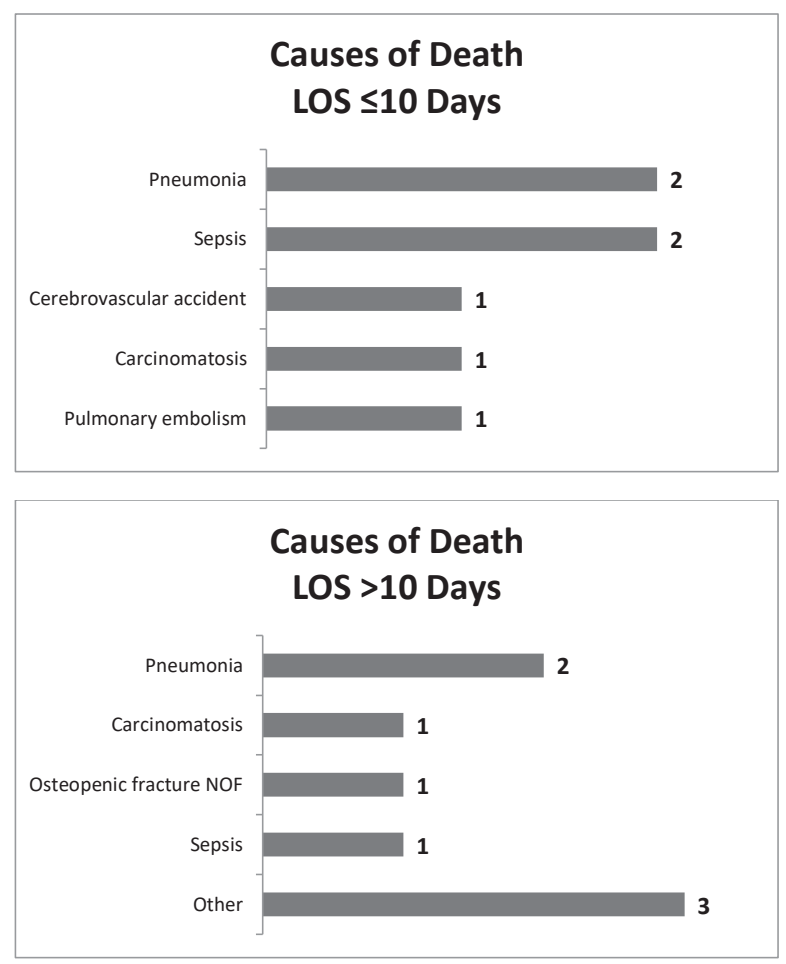

Figure 2. - Causes of Death at 30 Days Grouped by LOS. multiple co-morbidities in the mortality group. In both 30-day and 90-day mortality univariate analyses, admission from a source other than own home was also significant associated with mortality. However, there was no difference when LOS (10 days or less compared with more than 10 days) was analysed against 90-day mortality on univariate analysis.

The hazard ratio (HR) for mortality within 30days while having a LOS of $<10$ days after adjusting for covariates was 7.26 (95\% CI: 2.57-20.52). Other significant risk factors associated with 30-day mortality included increasing age, male gender and presence of concurrent chest infection during the index admission (Table 5). Following multivariate analysis adjusting for covariates, significant risk factors associated with 90-day mortality included increasing age, admission source other than own home, increasing ASA grade and a diagnosis of chronic obstructive airways disease (COAD - Table 6).

\section{DISCUSSION}

In our study cohort of 1228 patients treated for a fragility hip fracture, the mean LOS stay was 24 days. $16.8 \%$ of these patients had a LOS stay shorter than 10 days. After controlling for all significant covariates, a reduced LOS of less than 10 days was associated with 30-day mortality with a HR estimate of 7.26 (95\% CI: 2.57-20.52). Other risk factors of 30-day mortality included increasing age, male gender and concurrent active chest infection.

Reduced LOS was not associated with increased 90-day mortality. While increasing age was a common risk factor for both 30 and 90-day mortality, risk factors for 90-day mortality included admission source other than own home (mainly admission from nursing homes), increasing ASA grade and COAD. These are likely surrogate markers of chronic comorbidities which are expected to increase 90-day mortality. In contrast, a shorter LOS did not increase the risk of 90-day mortality suggesting that any detrimental effect of a shorter LOS is short-lived and does not extend to 90 days. This effect may be explained if patients discharged earlier were more infirm and had a higher risk of early mortality. For 
Table 1. - Patient demographics grouped by 30 day mortality status

\begin{tabular}{|c|c|c|c|}
\hline & $\begin{array}{l}\text { 30-Day Mortality } \\
\text { Group }(n=15)\end{array}$ & $\begin{array}{l}\text { 30-Day Survivor } \\
\text { Group }(n=1213)\end{array}$ & p-value \\
\hline $\begin{array}{l}\text { Gender } \\
\text { Male } \\
\text { Female }\end{array}$ & $\begin{array}{l}8 \\
7\end{array}$ & $\begin{array}{l}312 \\
901\end{array}$ & 0.02 \\
\hline Age in Years & 86 & 81 & 0.05 \\
\hline $\begin{array}{l}\text { Fracture Type } \\
\text { Intracapsular - displaced } \\
\text { Intracapsular - undisplaced } \\
\text { Intertrochanteric } \\
\text { Subtrochanteric } \\
\text { Other }\end{array}$ & $\begin{array}{l}3 \\
6 \\
4 \\
0 \\
2\end{array}$ & $\begin{array}{c}384 \\
371 \\
292 \\
94 \\
72\end{array}$ & 0.46 \\
\hline \begin{tabular}{|l} 
ASA grade \\
I \\
II \\
III \\
IV \\
V
\end{tabular} & $\begin{array}{l}2 \\
5 \\
5 \\
3 \\
0\end{array}$ & $\begin{array}{c}105 \\
334 \\
630 \\
143 \\
1\end{array}$ & 0.66 \\
\hline $\begin{array}{l}\text { Admission Source } \\
\text { Own home } \\
\text { Residential/Nursing home } \\
\text { Already inpatient in hospital } \\
\text { Inpatient from other hospital } \\
\text { Unknown }\end{array}$ & $\begin{array}{l}7 \\
7 \\
0 \\
0 \\
1\end{array}$ & $\begin{array}{c}956 \\
200 \\
28 \\
3 \\
26\end{array}$ & 0.02 \\
\hline $\begin{array}{l}\text { Pre-Injury Walking Ability } \\
\text { Independent } \\
\text { Single walking stick } \\
2 \text { sticks/frame } \\
\text { Wheelchair/scooter } \\
\text { Unknown }\end{array}$ & $\begin{array}{l}8 \\
3 \\
3 \\
0 \\
1\end{array}$ & $\begin{array}{c}657 \\
289 \\
211 \\
24 \\
32\end{array}$ & 0.85 \\
\hline Proportion of patients achieving surgery within 36 hours of admission & $10 / 15$ & $473 / 1213$ & 0.03 \\
\hline $\begin{array}{r}\text { Length of Stay } \\
\leq 10 \text { days } \\
>10 \text { days }\end{array}$ & $\begin{array}{l}7 \\
8\end{array}$ & $\begin{array}{c}199 \\
1014\end{array}$ & 0.01 \\
\hline
\end{tabular}

example if a patients were discharged to a nursing home because of dependence needs/co-morbidities and also because a nursing home placement (where rehabilitation can be ongoing) was possible to arrange quicker than fully rehabilitating a patient in hospital prior to discharge to their own home.

There are multiple confounding risk factors that may influence early mortality following hip fracture. Whilst our results show an association between short LOS and a higher risk of 30-day mortality, there are risk factors that we could not measure objectively that also influence early mortality. These include generalised frailty, independence with self-caring, malnutrition and patients naturally nearing the end of their life. Whilst our data contains surrogate markers for these risk factors (ASA grade, blood test results, walking ability etc.), it is almost impossible to quantify the effects of such risk factors directly.

A steady downward trend in early mortality rates has been seen in the management of hip fractures, owing to implementation of efficient perioperative processes such as fast track care pathways, multidisciplinary management and advanced rehabilitation protocols (17). However authors have recently also questioned whether a plateau has been reached in further improving early mortality from fragility 
Table 2. - Admission comorbidities and blood test results grouped by 30 day mortality status

\begin{tabular}{|c|c|c|c|}
\hline Variable & $\begin{array}{l}\text { 30-Day Mortality } \\
\text { Group }(n=15)\end{array}$ & $\begin{array}{c}\text { 30-Day Survivor Group } \\
(\mathrm{n}=1213)\end{array}$ & p-value \\
\hline \multicolumn{4}{|l|}{ Co-Morbidities on Admission } \\
\hline Mean Charlson Score & $5.1(4-8)$ & $4.4(0-9)$ & 0.05 \\
\hline Hypertension & 1 & 29 & 0.31 \\
\hline COAD & 3 & 76 & 0.08 \\
\hline Previous Stroke & 1 & 36 & 0.37 \\
\hline Previous Myocardial Infarction & 0 & 27 & 0.72 \\
\hline Chest infection during admission & 4 & 101 & 0.03 \\
\hline Chronic Heart Failure & 0 & 28 & 0.71 \\
\hline Chronic Liver Disease & 0 & 4 & 0.95 \\
\hline Dementia & 1 & 86 & 0.71 \\
\hline Diabetes Mellitus & 1 & 124 & 0.54 \\
\hline Neurological Disease & 0 & 26 & 0.72 \\
\hline Thyroid Disease & 2 & 71 & 0.22 \\
\hline Malignancy & 2 & 111 & 0.41 \\
\hline Alcohol Excess & 0 & 39 & 0.62 \\
\hline Urinary Tract Infection & 1 & 196 & 0.28 \\
\hline Peripheral Vascular Disease & 0 & 18 & 0.80 \\
\hline Cardiovascular Disease & 1 & 25 & 0.28 \\
\hline Connective Tissue Disorder & 0 & 1 & 0.99 \\
\hline Peptic Ulcer Disease & 0 & 8 & 0.91 \\
\hline Chronic Kidney Disease & 3 & 160 & 0.08 \\
\hline Hemiplegia & 0 & 28 & 0.84 \\
\hline Leukaemia & 0 & 8 & 0.95 \\
\hline \multicolumn{4}{|l|}{ Mean Blood Parameters on Admission } \\
\hline Haemoglobin $(10 \mathrm{~g} / \mathrm{dL})$ & 11.9 & 12.0 & 0.80 \\
\hline White Cell Count (X 109/li) & 11.1 & 10.7 & 0.74 \\
\hline Platelet Count (X 109/1i) & 248 & 282 & 0.26 \\
\hline Sodium (mmol/li) & 138 & 137 & 0.27 \\
\hline Potassium (mmol/li) & 4.3 & 4.3 & 0.99 \\
\hline Urea $(\mathrm{mmol} / \mathrm{li})$ & 10.0 & 8.1 & 0.07 \\
\hline Creatinine $(\mu \mathrm{mol} / \mathrm{li})$ & 120 & 101 & 0.09 \\
\hline INR & 1.2 & 1.1 & 0.52 \\
\hline APTT & 31.9 & 30.4 & 0.51 \\
\hline
\end{tabular}

hip fracture management (17). Perhaps this may be attributed to the fact that pre-operative risk factors for mortality in such patients are largely non modifiable. The findings of a meta-analysis of over 75 hip fracture studies have confirmed patient level preoperative risk factors such as increasing age and male gender to be strong risk factors for early postoperative mortality following 
Table 3. - Patient demographics grouped by 90 day mortality status

\begin{tabular}{|c|c|c|c|}
\hline & $\begin{array}{l}\text { 90-Day Mortality } \\
\text { Group }(\mathrm{n}=96)\end{array}$ & $\begin{array}{l}\text { 90-Day Survivor } \\
\text { Group (n=1132) }\end{array}$ & p-value \\
\hline $\begin{array}{c}\text { Gender } \\
\text { Male } \\
\text { Female }\end{array}$ & $\begin{array}{l}30 \\
66\end{array}$ & $\begin{array}{l}290 \\
842\end{array}$ & 0.23 \\
\hline Age in Years & 86.0 & 80.4 & $<0.01$ \\
\hline $\begin{array}{l}\text { Fracture Type } \\
\text { Intracapsular - displaced } \\
\text { Intracapsular - undisplaced } \\
\text { Intertrochanteric } \\
\text { Subtrochanteric } \\
\text { Other }\end{array}$ & $\begin{array}{c}25 \\
28 \\
31 \\
7 \\
5\end{array}$ & $\begin{array}{c}362 \\
349 \\
265 \\
87 \\
69\end{array}$ & 0.39 \\
\hline \begin{tabular}{|c} 
ASA grade \\
I \\
II \\
III \\
IV \\
V
\end{tabular} & $\begin{array}{c}3 \\
15 \\
58 \\
20 \\
0\end{array}$ & $\begin{array}{c}104 \\
324 \\
577 \\
126 \\
1\end{array}$ & $<0.01$ \\
\hline $\begin{array}{l}\text { Admission Source } \\
\text { Own home } \\
\text { Residential/Nursing home } \\
\text { Already inpatient in hospital } \\
\text { Inpatient from other hospital } \\
\text { Unknown }\end{array}$ & $\begin{array}{c}57 \\
32 \\
3 \\
1 \\
3\end{array}$ & $\begin{array}{c}906 \\
175 \\
25 \\
2 \\
24\end{array}$ & $<0.01$ \\
\hline \begin{tabular}{|l} 
Pre-Injury Walking Ability \\
Independent \\
Single walking stick \\
2 sticks/frame \\
Wheelchair/scooter \\
Unknown
\end{tabular} & $\begin{array}{c}44 \\
18 \\
27 \\
2 \\
5\end{array}$ & $\begin{array}{c}621 \\
274 \\
187 \\
22 \\
28\end{array}$ & 0.02 \\
\hline Proportion of patients achieving surgery within 36 hours of admission & $41 / 55$ & $442 / 690$ & 0.27 \\
\hline $\begin{array}{r}\text { Length of Stay } \\
\leq 10 \text { days } \\
>10 \text { days }\end{array}$ & $\begin{array}{l}18 \\
78\end{array}$ & $\begin{array}{l}188 \\
944\end{array}$ & 0.33 \\
\hline
\end{tabular}

hip fracture surgery (18) which is in keeping with the findings of our study. The same study also identified a higher ASA grade and the presence of multiple comorbidities to be strong risk factors of early mortality which is also in agreement with our findings.

Our reported mortality in this cohort is low due to the fact that we only included patients who died after being discharged from their index, hip fracture admission. This figure is explained by the fact that patients who died as inpatients were excluded (a total of 128 patients). These patients could not be included in the analysis as the purpose of the study was ultimately to analyse whether patients were being discharged too early.

Our results are similar to the findings of the recent Swedish study (11) which showed that a LOS of 10 days or less is independently associated with an increased risk of 30-day mortality in their hip fracture population. The authors showed that early discharge was associated with dementia, a risk factors that we have controlled for when calculating the risk of mortality. This suggests that short LOS may be related to poor overall health status and a shorter life expectancy. The findings of our study are consistent with that of Kondo et al. (12) who in their 
Table 4. - Admission comorbidities and blood test results grouped by 90 day mortality statusa

\begin{tabular}{|c|c|c|c|}
\hline Variable & $\begin{array}{l}\text { 90-Day Mortality } \\
\text { Group }(n=96)\end{array}$ & $\begin{array}{l}\text { 90-Day Survivor Group } \\
(\mathrm{n}=1132)\end{array}$ & p-value \\
\hline \multicolumn{4}{|l|}{ Co-Morbidities on Admission } \\
\hline Mean Charlson Score & 5.0 & 4.4 & $<0.01$ \\
\hline Hypertension & 5 & 25 & 0.08 \\
\hline COAD & 15 & 64 & 0.00 \\
\hline Previous Stroke & 5 & 32 & 0.16 \\
\hline Previous Myocardial Infarction & 3 & 24 & 0.35 \\
\hline Chest infection during admission & 13 & 92 & 0.06 \\
\hline Chronic Heart Failure & 2 & 26 & 0.62 \\
\hline Chronic Liver Disease & 0 & 4 & 0.72 \\
\hline Dementia & 14 & 73 & 0.01 \\
\hline Diabetes Mellitus & 10 & 115 & 0.52 \\
\hline Neurological Disease & 1 & 25 & 0.38 \\
\hline Thyroid Disease & 6 & 67 & 0.51 \\
\hline Malignancy & 12 & 101 & 0.16 \\
\hline Alcohol Excess & 1 & 38 & 0.18 \\
\hline Urinary Tract Infection & 12 & 185 & 0.20 \\
\hline Peripheral Vascular Disease & 2 & 16 & 0.42 \\
\hline Cardiovascular Disease & 4 & 22 & 0.14 \\
\hline Connective Tissue Disorder & 0 & 1 & 0.92 \\
\hline Peptic Ulcer Disease & 1 & 7 & 0.48 \\
\hline Chronic Kidney Disease & 13 & 70 & 0.01 \\
\hline Hemiplegia & 0 & 14 & 0.32 \\
\hline Leukaemia & 0 & 4 & 0.72 \\
\hline \multicolumn{4}{|l|}{ Mean Blood Parameters on Admission } \\
\hline Haemoglobin $(10 \mathrm{~g} / \mathrm{dL})$ & 11.7 & 12.0 & 0.04 \\
\hline White Cell Count (X 10\%/li) & 11.2 & 10.7 & 0.23 \\
\hline Platelet Count (X 10\%/li) & 275 & 281 & 0.58 \\
\hline Sodium (mmol/li) & 138 & 137 & 0.08 \\
\hline Potassium (mmol/li) & 4.4 & 4.3 & 0.45 \\
\hline Urea $(\mathrm{mmol} / \mathrm{li})$ & 9.4 & 8.0 & 0.08 \\
\hline Creatinine $(\mu \mathrm{mol} / \mathrm{li})$ & 110 & 100 & 0.00 \\
\hline INR & 1.1 & 1.1 & 0.16 \\
\hline APTT & 30.0 & 30.5 & 0.35 \\
\hline
\end{tabular}

comparative study showed favourable survival rates of $89.5 \%$ in fragility hip fracture patients treated in Japanese healthcare institutions where the median LOS was 34 days compared to $77.2 \%$ in patients from American hospitals with a median LOS of 5 days. In contrast, a more recent American study by Nikkel et al. from the state of New York has shown a $32 \%$ increased odds of 30-day mortality in hip fracture patients when LOS increased beyond 10 days (19). 
Table 5. - Multivariate analysis for 30 day mortality

\begin{tabular}{|l|c|c|c|c|}
\hline \multirow{2}{*}{ Variable } & \multirow{2}{*}{ p-value } & \multirow{2}{*}{ Hazard Ratio } & \multicolumn{2}{|c|}{ Confidence Interval (95\%) } \\
\cline { 3 - 5 } & & & Lower & Upper \\
\hline Increasing Age & 0.00 & 1.11 & 1.03 & 1.19 \\
\hline Chest Infection During Admission & 0.02 & 3.98 & 1.25 & 12.67 \\
\hline Male Gender & 0.01 & 4.18 & 1.49 & 11.77 \\
\hline Length of Stay of 10 days or less & $<0.01$ & 7.26 & 2.57 & 20.52 \\
\hline
\end{tabular}

Table 6. - Multivariate analysis for 90 day mortality

\begin{tabular}{|l|c|c|c|c|}
\hline \multirow{2}{*}{ Variable } & \multirow{2}{*}{ p-value } & \multirow{2}{*}{ Hazard Ratio } & \multicolumn{2}{c|}{ Confidence Interval (95\%) } \\
\cline { 4 - 5 } & & & Lower & Upper \\
\hline Increasing Age & $<0.01$ & 1.07 & 1.04 & 1.10 \\
\hline Admission source other than own home & 0.04 & 1.24 & 1.02 & 1.53 \\
\hline Increasing ASA grade & 0.01 & 1.45 & 1.09 & 1.93 \\
\hline COAD & $<0.01$ & 2.76 & 1.56 & 4.89 \\
\hline
\end{tabular}

Early mortality after fragility hip fractures is generally attributed to patient factors and to assume a direct causal relationship between LOS and mortality maybe too simplistic an approach. Discharge destinations from hospital, within the American healthcare system are different to that of its European and Asian counterparts. In the US $90 \%$ of fragility hip fracture patients have access to rehabilitation facilities post operatively where much of their follow up care is undertaken (20). Discharge from hospital to focussed rehabilitation facilities which serve a transitional role prior to a more permanent destination may help prevent early mortality (21). In contrast, more patients are discharged home or to a final destination in England and other European countries (22). It is hence likely that greater LOS in the UK and other European healthcare facilities where majority of the postoperative care and rehabilitation is undertaken in the same hospital episode, is likely to be associated with favourable outcomes and lower mortality as suggested by our results. The difference in standards of care between American and British or European healthcare providers with respect to fragility hip fracture management during the inpatient episode may also be a factor in contributing to the relationship of LOS and early mortality. Certainly within the UK, hip fracture guidelines advocate surgery within
36 hours of presentation and healthcare institutions are financially incentivised to do so (23). Similarly standards of care within Swedish healthcare systems advocate hip fracture stabilisation within 24 hours of presentation for nearly all patients (24). In contrast Nikkel et al. in their American state wide study showed that $19.4 \%$ of patients waited more than two days for their surgery and such delay to surgery was associated with increased LOS (19). It is therefore possible that their findings of increased LOS being associated with early mortality may actually represent a latent relationship of surgical delay and early mortality which is well established.

In the United Kingdom, following admission and surgery for a hip fracture the focus is on rehabilitating the patient to a point where they can be safely discharged to the community or a centre with supported rehabilitation. This is based on national guidelines (15) and led by a multidisciplinary team providing daily care to the patient. It is usually assumed that in a developed Western healthcare system, a patient will be kept admitted as long as their clinical condition and rehabilitation requires them to be. However, multiple factors contribute to increasing bed pressures within the NHS that including limited funding, an ageing population requiring more inpatient care and a relative lack of resources. The national focus is on reducing LOS by 
using strategies such as discharge to physiotherapy led rehabilitation unit or supported accommodation and indeed, overall LOS in hip fracture admissions is decreasing (25). No doubt the trend is the same at our unit.

Our study has several strengths including a large overall cohort of patients reporting this outcome. Additionally, we report this finding that hasn't previously been reported in a UK population to the best of our knowledge. We were also able to report upon the causes of early mortality in this cohort that is often not possible with large registry level data. Finally, we included all co-morbidities and blood test findings in our analyses of mortality to minimise the confounding bias that may be included when analysing causes of mortality.

We recognise a limitation of our study in that its retrospective nature lacks the robustness of prospective data collection and analysis. However, all data is input prospectively by specially trained staff at our institute and we cross-checked multiple hospital databases to ensure its accuracy. Additionally, this method of data collection and analysis allowed for a large sample to be collected with comprehensive data on inpatient admission and mortality. It has been shown that the overall geriatric profile (e.g. as assessed by the Clinical Frailty Scale) itself carries a higher risk of complications and mortality following hip fracture (26) - this is a potential confounder that we could not account for in our analysis. Additionally we did analyse for specific geriatric syndromes such as acute delirium and end-of-life status as these data were not available to us. Another limitation was the significantly smaller 30-day mortality subgroup due to the inclusion of outpatient mortality only. This small number of events is potentially susceptible to bias at the patient level. Nevertheless, the exclusion of inpatient mortality was necessary to properly analyse the effect of LOS and the subgroup analysis remained statistically significant.

\section{CONCLUSION}

In conclusion our results seem to support the hypothesis that a shorter LOS following a hip fracture is associated with an increased risk of 30-day but not 90-day mortality in the UK hip fracture population. This association is likely to be multifactorial and not directly causal. We suggest a comprehensive rehabilitation regimen following admission with a fragility hip fracture may help mitigate this risk.

\section{REFERENCES}

1. Cooper C, Cole ZA, Holroyd CR, Earl SC, Harvey NC, Dennison EM, et al. Secular trends in the incidence of hip and other osteoporotic fractures. Osteoporos Int. 2011; 22(5): 1277-88.

2. Leal J, Gray AM, Prieto-Alhambra D, Arden NK, Cooper C, Javaid MK, et al. Impact of hip fracture on hospital care costs: a population-based study. Osteoporos Int. 2016; 27(2): 549-58.

3. Heggestad T. Do hospital length of stay and staffing ratio affect elderly patients' risk of readmission? A nation-wide study of Norwegian hospitals. Health Serv Res. 2002; 37(3): 647-65.

4. Aujesky D, Stone RA, Kim S, Crick EJ, Fine MJ. Length of hospital stay and postdischarge mortality in patients with pulmonary embolism: a statewide perspective. Arch Gen Intern Med. 2008; 168(7): 706-12.

5. Southern WN, Arnsten JH. Increased Risk of Mortality among Patients Cared for by Physicians with Short Lengthof-Stay Tendencies. J Gen Intern Med. 2015; 30(6): 712-8.

6. Chatterton BD, Moores TS, Ahmad S, Cattell A, Roberts PJ. Cause of death and factors associated with early in-hospital mortality after hip fracture. Bone Joint $J$. 2015; 97-B(2): 246-51.

7. Khan MA, Hossain FS, Ahmed I, Muthukumar N, Mohsen A. Predictors of early mortality after hip fracture surgery. Int Orthop. 2013; 37(11): 2119-24.

8. Sheikh HQ, Hossain FS, Aqil A, Akinbamijo B, Mushtaq V, Kapoor H. A Comprehensive Analysis of the Causes and Predictors of 30-Day Mortality Following Hip Fracture Surgery. Clin Orthop Surg. 2017; 9(1): 10-8.

9. Aqil A, Hossain F, Sheikh H, Aderinto J, Whitwell G, Kapoor H. Achieving hip fracture surgery within 36 hours: an investigation of risk factors to surgical delay and recommendations for practice. J Orthop Trauma. 2016; 17(3): 207-13.

10. Tsang C, Boulton C, Burgon V, Johansen A, Wakeman R, Cromwell DA. Predicting 30-day mortality after hip fracture surgery: Evaluation of the National Hip Fracture Database case-mix adjustment model. Bone Joint Res. 2017; 6(9): 550-6.

11. Nordstrom P, Gustafson Y, Michaelsson K, Nordstrom A. Length of hospital stay after hip fracture and short term risk of death after discharge: a total cohort study in Sweden. BMJ. 2015; 350: h696.

12. Kondo A, Zierler BK, Isokawa Y, Hagino H, Ito Y, Richerson M. Comparison of lengths of hospital stay after surgery and mortality in elderly hip fracture patients between Japan and the United States - the relationship 
between the lengths of hospital stay after surgery and mortality. Disabil Rehabil. 2010; 32(10): 826-35.

13. BBC News. We've not got enough beds or staff - NHS bosses 2018 [February 2020]. Available from: https://www. bbc.com/news/health-43576036.

14. Charlson ME, Pompei P, Ales KL, MacKenzie CR. A new method of classifying prognostic comorbidity in longitudinal studies: development and validation. J Chronic Dis. 1987; 40(5): 373-83.

15. National Institute of Health and Care Excellence. Hip fracture: management 2017 [February 2019]. Available from: https://www.nice.org.uk/guidance/cg124.

16. Bursac Z, Gauss CH, Williams DK, Hosmer DW. Purposeful selection of variables in logistic regression. Source Code Biol Med. 2008; 3: 17.

17. Giannoulis D, Calori GM, Giannoudis PV. Thirty-day mortality after hip fractures: has anything changed? Eur $J$ Orthop Surg Traumatol. 2016; 26(4): 365-70.

18. Hu F, Jiang C, Shen J, Tang P, Wang Y. Preoperative predictors for mortality following hip fracture surgery: a systematic review and meta-analysis. Injury. 2012; 43(6): 676-85.

19. Nikkel LE, Kates SL, Schreck M, Maceroli M, Mahmood B, Elfar JC. Length of hospital stay after hip fracture and risk of early mortality after discharge in New York state: retrospective cohort study. BMJ. 2015; 351: h6246.

20. Goodman D, Fisher E, Chang C. After Hospitalization: A Dartmouth Atlas Report on Post-Acute Care for Medicare Beneficiaries 2011 [March 2020]. Available from: https://
www.dartmouthatlas.org/downloads/reports/Post discharge_events_092811.pdf.

21. Lahtinen A, Leppilahti J, Harmainen S, Sipila J, Antikainen R, Seppanen ML, et al. Geriatric and physically oriented rehabilitation improves the ability of independent living and physical rehabilitation reduces mortality: a randomised comparison of 538 patients. Clin Rehab. 2015; 29(9): 892-906.

22. Royal College of Physicians. National Hip Fracture Database (NHFD) Annual Report 20162016 [March 2020]. Available from: https://www.nhfd.co.uk/20/hipfractureR. nsf/docs/reports2016.

23. Metcalfe D, Zogg CK, Judge A, Perry DC, Gabbe B, Willett K, et al. Pay for performance and hip fracture outcomes: an interrupted time series and difference-indifferences analysis in England and Scotland. Bone Joint J. 2019; 101-B(8): 1015-23.

24. Ahman R, Siverhall PF, Snygg J, Fredrikson M, Enlund G, Bjornstrom K, et al. Determinants of mortality after hip fracture surgery in Sweden: a registry-based retrospective cohort study. Scientific reports. 2018; 8(1): 15695.

25. Kumar G. Protocol-guided hip fracture management reduces length of hospital stay. Br J Hosp Med. 2012; 73(11): 645-8.

26. Chen C-L, Chen C-M, Wang C-Y, Ko P-W, Chen C-H, Hsieh C-P, et al. Frailty is Associated with an Increased Risk of Major Adverse Outcomes in Elderly Patients Following Surgical Treatment of Hip Fracture. Scientific Rep. 2019; 9(1): 19135. 